\title{
The design requirements of a comprehensive nutrition and dietetic portal based on the experiences of selected countries
}

\author{
Zahra Sadat Abtahi ${ }^{1, *}$, Reza Safdari ${ }^{2}$, Majan Ghazi Saeedi ${ }^{2}$, Ahmad Reza Dorosty $^{3}$ \\ ${ }^{1}$ Tehran University of Medical Sciences, School of Allied Medical Sciences \\ ${ }^{2}$ Tehran University of Medical Sciences, School of Allied Medical Sciences, Department of Health Information Management \\ ${ }^{3}$ Tehran University of Medical Sciences, School of Nutritional Sciences and Dietetics, Department of Nutrition
}

Email address:

Zahra_abtahi65@yahoo.com (Z.S. Abtahi)

\section{To cite this article:}

Zahra Sadat Abtahi, Reza Safdari, Majan Ghazi Saeedi, Ahmad Reza Dorosty. The Design Requirements of a Comprehensive Nutrition and Dietetic Portal Based on the Experiences of Selected Countries. International Journal of Nutrition and Food Sciences.

Vol. 4, No. 2, 2015, pp. 132-136. doi: 10.11648/j.ijnfs.20150402.12

\begin{abstract}
Background and Objectives: Undoubtedly, information and communication technology resulted in a massive development in all social and economic areas. It has a great impact on human communities so that the world is rapidly becoming an information society. It is vital to find additional ways to reduce overweight due to increased rate of obesity and obesity-related health problems. Nutrition and dietetic portals provide access to a wide range of information resources for nutritionists, physicians and the public due to the dynamic content and integrated access to resources, software, applications and databases. The aim of the present study is to review the nutrition and dietetic portals in selected countries to provide some suggestions in this field. Methods: This is a descriptive-comparative study. The minimum content features of the nutrition and dietetic portals were identified through comprehensive review of the literature. The nutrition and dietetic portals of the selected countries were examined through observation, communication and completion of a checklist. The second stage involved the design of a dietetic system. Accordingly, a HTML-based (Hyper Text Markup Language) system was designed using the semiexperimental study. Results: The common aspects of the nutrition and dietetic portals include annual statistics, nutrition news, calendar of events, information on job opportunities, information on different diets, strategies and policies, professional standards and rules, nutrition guidelines and manuals as well as educational and research facilities. Conclusion: To provide accessible information for the public and healthcare providers, the design of a specialized comprehensive portal with desirable specifications and a credible scientific background will have a significant impact on the promotion of nutrition literacy and thereby improved heath of society through analyzing the users' needs.
\end{abstract}

Keywords: Nutrition, Diet, Portal, Health Information Technology

\section{Introduction}

Food and nutrition are among the most essential human needs from the first moment to the last moment of life [1]. Food provides energy and countless materials required for the construction of the human body and for its growth and survival [2].The nutritional habits of every nation indicate the international economic and political values of that nation [3]. The development of modern biological sciences and technologies revealed that the growth and development of the human body as well as scrutiny and mind dynamism are closely related to type and quality of diet. The nutritional habit has a great impact on health and diseases in some periods of evolution and growth. Accordingly, the lack of a balanced and varied diet will cause some irreparable side effects. Thus, one of the most important concerns of learned scholars and sympathetic policymakers is to promote the culture of appropriate and balanced nutrition throughout the world [2]. It is vital to find additional ways to reduce overweight due to increased rate of obesity and obesityrelated health problems .According to Saperstein et al., the number of people who use Internet to find information about diet, physical activity and weight loss is increasing.

The online applications help participants to give priority to 
their diets to reduce their weights. Such applications also help the users to change their physical activities and to apply behavioral and cognitive strategies for individual supervision and feedback from their diets. In addition, the online applications increase media literacy of the public [4]. Accordingly, one of the best solutions to solve this problem is to use web portal technology. In Information Technology (IT), the portal refers to a website in the Internet or intranet as a gateway to access other websites [5].

The obstacles resulting from time constraints can be eliminated through establishing a portal to provide access to a comprehensive suite of electronic resources and services related to a specific field. Consequently, the search process of the technical resources is accelerated and therefore the search time is reduced. On the other hand, the portals are informational contexts to provide the required applications or software for e-business on the web. Furthermore, the portals integrate scattered information of databases or websites, regardless of the location and format through dynamic links [6]

With development of health portals as the most important technical infrastructure for e-health in various countries, the specialized health portals including nutrition and dietetic portals were also established. Nutrition and dietetic portals will enhance the nutrition literacy and knowledge of the public and nutritionists through dynamic content and integrated access to resources, software, applications, databases, content customization, personalization, navigation and interactive or collaborative services [7].

Therefore, the attempts to establish and operate the nutrition portal in Iran aim at developing informatics activities [9]. In this regard, taking advantage of the experiences on the operation of international nutrition and dietetic portals is necessary for benchmarking their characteristics. Accordingly, the present study reviews the content and structural characteristics of the nutrition portals in selected countries. Finally, several suggestions are presented in this regard.

\section{Methods}

This is across-sectional descriptive-comparative study conducted in 2014. In the first stage, the minimum content features of the nutrition and dietetic portals were identified through systematic study of literature and parameters in previous studies on nutrition and diet. The extracted features were included in a checklist containing 9 sections and 42 parameters. The content validity of the checklist was approved by the professors. The sample consisted of American, Canadian and Australian nutrition and dietetic portals. Then, the predetermined parameters were studied through observation and completion of the checklist. In the case where the portal content or some parameters were not available for security reasons, the researcher sent the checklist to the portal's officials to receive the required information .In the second stage, the HTML-based Iranian Nutrition and Dietetic Portal was designed and developed through semi-experimental study (www.hbisa.org/nutrition).
Finally, several strategies were suggested to develop a national nutrition and dietetic portal in Iran.

\section{Results}

The results show that the national nutrition and dietetic portals have been established and developed in USA, Canada and Australia. Tables 1 and 2 show the comparative analysis of the content features of the nutrition and dietetic portals.

\section{1. $U S A$}

The results showed that the U.S. health care system is a combination of public and private sectors [8]. The first department related to the agriculture and nutrition was US Department of Agriculture "USDA". In 1862, President Abraham Lincoln approved a bill concerning the establishment of USDA. This department was created to become a state scientific organization [9].

American Dietetic Portal is affiliated to the American Dietetic Association (ADA) as the world's largest organization of food and nutrition science. The Association is committed to improve public health and nutrition profession through education, research and support. The Association Board of Directors includes national leaders in the field of food, nutrition and health. ADA aims at optimizing the public health through food and nutrition. The mission of ADA is to empower the members to create a national system of food and nutrition [10].

Another active portal in the USA is the American Society for Nutrition (ASN). ASN is a non-profit organization which brings together the top researchers as well as clinical and industrial nutritionists to contribute to the advancement of the knowledge of human and animal nutrition. Currently, the Association focuses on the clinical research. The mission of ASN is to develop the knowledge of nutrition through basic, clinical and multidisciplinary research, facilitating communication between doctors and nutritionists and those interested in the related areas, supporting the development and application of nutrition science to improve the clinical and general heath throughout the world, promoting the medical education in nutrition and providing reliable nutritional information to those who need such information [11].

Another portal studied in USA is the American Dietetic Association. ADA is a non-profit organization which is exempt from tax. The mission of ADA is to promote the optimal health through nutrition and health education. ADA is a scientific association to provide professionals and the public with information on the benefits of proper nutrition and health [12].

The results suggest that the American nutrition and dietetic portals contain general information such as news and events related to nutrition and diet, job opportunities for dietitians and students, calendar of events, annual statistics, information on healthy weight, weight loss and weight gain. The portals also provide diets for infants and toddlers, children and adolescents, nutrition in school, women's nutrition during pregnancy, lactation, bone and heart health, 
nutrition during menopause, men's nutrition for active life, elderly nutrition, nutrition in different seasons and different cultures, different food resources in Chinese and Spanish cultures, vegetarian diet and electronic newsletters.

The technical content of the portals include policies, nutrition support service, professional standards and rules. The American portals uniquely provide services such as remote health services, rich foods. The audiences of the portals are nutritionists and the public. The educational content of the portals include online conferences, annual scientific meetings, remote education and specialized forums on nutrition and diet. The portals provide access to books, newspapers, magazines, video library and E-book shopping $[10,11,12]$.

\subsection{Canada}

According to the results, the major part of healthcare services is provided for free by the public health care system. In addition, some services are provided by the private sector [13]. The council for development of diet standards was established in 1938 to develop the required standards. The Council considers food standards and the essential nutrients to meet the needs of all healthy individuals [14].

One of the main nutrition and dietetic portals in Canada is Dietitians of Canada (DC). This is a professional association for nutritionists. DC aims at improving health through food and nutrition. Its mission is to support ethics and the best evidence-based diet and increase the health in a unique manner using the knowledge of food and nutrition, supporting members in their varied and rewarding roles as the leaders promoting health, lifelong education as well as training dietetic-related careers in Canada [15].

Among the active portals in Canada is Canadian Nutrition Society (CNS) established by merging the Canadian Association of Clinical Nutrition and Canadian Association of Nutrition Sciences. The mission of CNS is to raise nutrition knowledge and training, support the use of best practices and policies to promote health and prevent and treat diseases. CNS integrates the nutrition-related disciplines and professions. The Society has identified the key objectives and sub-objectives in one field: to help nutritionists and scientists, to help feed industry, state, community and trainees [16].

The portals provide both public and secure access platforms. The public platform provides access to the nutrition news, resources and job opportunities for dietitians, calendar of events, and basic tips for health promotion in different age groups and diseases, nutrition of infants, children and adolescents, nutrition in school, women's nutrition during pregnancy, sports nutrition, vegetarian diet and general nutritional information. The secure platform provides access to policies and food regulations, basic tools to support the work, self-assessment tools of nutritional status as well as professional standards and rules. Features such as nutrition in indigenous areas, remote health services, nutrition services for the elderly at home, nutrition and dietary services at long-term care centers and hospitals, labeling nutritional information and food fortification are unique features of the Canadian portal. The Canadian portal includes educational modules such as the national conferences, annual scientific meetings, online training and specialized nutrition and dietetic forums. The portal also provides online book and poster ordering, access to nutritional publications and magazines as well as research conducted in the field of nutrition $[15,16]$.

\subsection{Australia}

The results show that medical care services in Australia are provided by both public and private sectors. The Ministry of Health is the supreme institution monitoring and adopting health policies [17]. Interest in forming a scientific society for nutrition professionals in Australia began to grow in early 1971. The first step was taken by a small group of specialists at a meeting in Melbourne in March, 1974 leading to the formation of Nutrition Society of Australia (NSA) [18].

The Australian Nutrition Portal is a non-governmental, non-profit organization with several offices throughout Australia. NSA is an independent organization to promote the health and welfare of all Australians. The mission of the Society is to promote the health of all Australians through encouraging food variety and physical activities. NSA is managed by a national board which is responsible for taking strategic decisions and nutrition policies in Australia. The purposes of the Society as a credible source of scientific information on key issues of nutrition include the production and distribution of food among policymakers, media, trainers, consultants in government offices, the food industry and consumer groups on topics related to food and nutrition and encourage the dissemination of nutritional knowledge [19]. Another important portal in Australia is Dietitians Association of Australia (DAA). DAA is a national professional association with branches in all states and regions in Australia. DAA was founded by the Dietitians Association of Australia in 1976. The Society is a leader for better food, improved health and wellbeing for all Australians. As its mission, the Society provides the apex of professional diet and nutrition as well as strategic leadership in food and nutrition through empowerment, support, education, authentication and communication [20].

The results suggest that the public content of the Australian nutrition and dietetic portals include nutrition news, annual statistics, calendar of events, job opportunities, general nutrition, adult nutrition, nutrition for children and adolescents, elderly nutrition, sports nutrition, school nutrition, diets for various diseases, nutritional information and vegetarian diets. The portals also contain e-newsletters and media alerts. The unique features of the Australian nutrition portals include politics, healthy life pyramid, clinical guidelines, professional standards and rules, selfassessment software and labeling foods and various sports activities. The educational contents of the Australian portals include national conferences, annual scientific meetings, educational newsletters, lectures and slides. The portals also provide online book, poster and pamphlet ordering, evidencebased practice in nutrition as well as diet books and 
magazines $[10,20]$.

Table 1. The content of nutrition and dietetic portals in selected countries.

\begin{tabular}{|c|c|c|c|c|}
\hline Features & & Australia & Canada & USA \\
\hline \multirow{15}{*}{ General content } & 1- News and events related to nutrition and diet & $\sqrt{ }$ & $\checkmark$ & $\sqrt{ }$ \\
\hline & 2- Job opportunities & $\checkmark$ & $\checkmark$ & $\checkmark$ \\
\hline & 3-Annual statistics & $\checkmark$ & - & $\checkmark$ \\
\hline & 4- Calendar of events & $\checkmark$ & $\checkmark$ & $\checkmark$ \\
\hline & 5- Vegetarian diet & $\checkmark$ & $\checkmark$ & $\checkmark$ \\
\hline & 6- Disease-related nutrition & $\checkmark$ & $\checkmark$ & $\checkmark$ \\
\hline & 7- Nutrition for infants, children and adolescents & $\checkmark$ & $\checkmark$ & $\checkmark$ \\
\hline & 8- Nutrition in school & $\checkmark$ & $\checkmark$ & $\checkmark$ \\
\hline & 9- Women's nutrition in different periods & - & $\checkmark$ & $\checkmark$ \\
\hline & 10- Men's nutrition in different periods & - & - & $\checkmark$ \\
\hline & 11- Elderly nutrition & $\checkmark$ & - & $\checkmark$ \\
\hline & 12- Sports nutrition & $\checkmark$ & $\checkmark$ & - \\
\hline & 13- Nutrition in different seasons & - & - & $\checkmark$ \\
\hline & 14- General nutritional information & $\checkmark$ & $\checkmark$ & - \\
\hline & 15- Electronic newsletter & $\checkmark$ & - & $\checkmark$ \\
\hline \multirow{11}{*}{ Specialized content } & 16- Policies and strategies & $\checkmark$ & $\checkmark$ & $\checkmark$ \\
\hline & 17- Support for nutritional services & - & $\checkmark$ & $\checkmark$ \\
\hline & 18- Instructions and guidelines on nutrition and diet & $\checkmark$ & $\checkmark$ & $\checkmark$ \\
\hline & 19- Professional standards and rules & $\checkmark$ & $\checkmark$ & $\checkmark$ \\
\hline & 20- Self-assessment softwarefor nutritional status & $\checkmark$ & $\checkmark$ & - \\
\hline & 21- Remote health services of nutritionists & - & $\checkmark$ & $\checkmark$ \\
\hline & 22- Nutrition services for the elderly at home & - & $\checkmark$ & - \\
\hline & 23- labeling foods in terms of nutritional information and value & $\checkmark$ & $\checkmark$ & - \\
\hline & 24- Food fortification & - & $\checkmark$ & $\checkmark$ \\
\hline & 25- Food pyramid & $\checkmark$ & - & $\checkmark$ \\
\hline & 26- Nutrition services in long term care centers and hospitals & - & $\checkmark$ & - \\
\hline
\end{tabular}

Table 2. The educational and research features and links of nutrition and dietetic portals.

\begin{tabular}{|c|c|c|c|c|}
\hline Features & & Australia & Canada & USA \\
\hline \multirow{7}{*}{$\begin{array}{l}\text { Educational content } \\
\text { and services }\end{array}$} & 1- News and events related to nutrition and diet & $\checkmark$ & $\checkmark$ & - \\
\hline & 2- Job opportunities & $\checkmark$ & $\checkmark$ & $\checkmark$ \\
\hline & 3-Annual statistics & $\checkmark$ & $\checkmark$ & $\checkmark$ \\
\hline & 4- Calendar of events & $\checkmark$ & $\checkmark$ & $\checkmark$ \\
\hline & 5- Vegetarian diet & $\checkmark$ & - & - \\
\hline & 6- Disease-related nutrition & $\checkmark$ & $\checkmark$ & - \\
\hline & 7- Nutrition for infants, children and adolescents & - & $\checkmark$ & $\checkmark$ \\
\hline \multirow{4}{*}{ Research content } & 8- Online training & $\checkmark$ & $\checkmark$ & $\checkmark$ \\
\hline & 9- Educational programs on nutrition and diet & - & - & $\checkmark$ \\
\hline & 10- National conferences & $\checkmark$ & - & - \\
\hline & 11- Annual scientific meetings & $\checkmark$ & $\checkmark$ & $\checkmark$ \\
\hline \multirow{5}{*}{$\begin{array}{l}\text { Dynamic links to } \\
\text { websites }\end{array}$} & 12- Department of Health and Human Services & $\checkmark$ & $\checkmark$ & $\checkmark$ \\
\hline & 13- Department of Agriculture & $\checkmark$ & $\checkmark$ & - \\
\hline & 14- Department of Food and Drug & $\checkmark$ & $\checkmark$ & $\checkmark$ \\
\hline & 15- Schools and institutions & - & $\checkmark$ & - \\
\hline & 16- health and nutrition associations and institutions & $\checkmark$ & $\checkmark$ & $\checkmark$ \\
\hline
\end{tabular}

\section{Discussion and Conclusion}

The results showed that web portal technology is considered as a part of e-health strategic plan in Canada due to its efficiency in integrating software packages, services and web-based data [21]. Shim and Sorrells described the portal development as a strategy for the success of e-health system [22]. The importance of web portal technology has been recognized in Iran. According to Torabi et al., the development of organizational portals is necessary to integrate activities in line with the implementation of e-health in Iran [23].

According to findings, the most common content of American, Canadian and Australian portals include statistics, news and calendar of events on diet and nutrition. In addition, all portals provide information on job opportunities [10, 15, 19]. Furthermore, all three portals provide information on nutrition and diet in disease and different periods of life [11, 16, 20]. However, only the American portal provides nutritional information for men and nutrition in different seasons [12]. The results showed that all portals provide information on policies, professional standards and instructions and guidelines related to nutrition and diet. However, nutrition support services, remote health services and food fortification are embedded only in the American and Canadian portals $[11,16]$. Nutrition services for the elderly at home and nutrition services in long-term care centers and hospitals are the unique features of the Canadian portal [15]. 
Training is the common aspects of nutrition and dietetic portals in the selected countries. However, online training and specialized nutrition forums are available only in the American and Canadian portals. Video library is of the items included only in the American portal. One of the most crucial aspects of the nutrition and dietetic portals is training services to professionals, students and the public. All portals provide access to books, newspapers, magazines, journals as well as online book ordering through online stores $[10,15,19]$.

The results indicated that the Canadian portal provides more comprehensive links to the relevant websites. The dynamic links to organizations and institutions is essential for a nutrition and dietetic portal. According to what discussed, it is necessary to design and establish a specialized portal with all desirable features and specifications with continuing analysis of the demands of end users with a credible scientific background. In addition, the main requirement for the integration of services and content offered by the portal is the presence of a defined and integrated structure of the entities in the field of nutrition and diet and health-related sectors. The results of the present study are a clear example of this fact.

Therefore, the following general measures seem necessary to establish a national nutrition and dietetic portal in Iran prior to considering the structural and content features:

1. Establishing and developing a national nutrition and dietetic portal supported by government agencies such as the Ministry of Health and Nutrition Association to provide access to information for the public and professionals.

2. Setting goals and expected benefits of the nutrition portal, portal users, the establishment and operating costs of the portal considering similar examples in other countries

3. Assessment of training, research, information and service needs of the portal users

4. To match the analyzed needs with current standards and policies

5. The conceptual, technical and visual design of the portal

6. Setting up and continuous improvement and update of the portal and training the users

\section{References}

[1] HSE-NPC Nutrition and Food Hygiene;Available from: http://khash.zaums.ac.ir/uploads/TAGHZIE_27593.pdf

[2] mahan, L.K., S. escott-stump, and J. raymond, krause's Food and the Nutrition Care Process, 2012(Book).

[3] hatami, H. and S.m. safavi, The Book of Public Health. 2004.

[4] Saperstein, S.L., N.L. Atkinson, and R.S. Gold, The impact of Internet use for weight loss. International Association for the Study of Obesity, 2007. 8: p. 459-465.
[5] Carbone, D. and S. Burgess, Health portals: An exploratory review. In: Tan J. Medical informatics:Concepts, methodologies, tools, and applications. Medical Information science Reference, 2009: p. 58-61.

[6] Tatnall, A., Web portals: The new gateways to Internet information and services, in Hershey.Idea Group Publishing2005. p. 3-7,6-22.

[7] safdari, r., et al., Achievements of Nursing Portal Development in Selected Countries. Journal of Nursing and Midwifery, Tehran University of Medical Sciences, 1390. 4(17): p. 46-62.

[8] IGLEHART, J.K., THE AMERICAN HEALTH CARE SYSTEM. Health Policy Report, 1999. 340.

[9] The Early History Of American Nutrition Reserach. 2009. ; Available from: http://www.sunypress.edu/pdf/61750.pdf

[10] Academy of Nutrition and Dietetics. 20/6/2013]; Available from: http://www.eatright.org/Media/content.aspx?id=6442467510.

[11] United States Department of Agriculture "Vegetarian Diet ". 26/5/2013]; Available from: http://www.nlm.nih.gov/medlineplus/vegetariandiet.html.

[12] THE BRITISH DIETETIC ASSOCIATION "Vegetarian diets - Keeping a healthy balance."

[13] Health care in Canada. 2013 1/1/2014]; Available from: http://en.wikipedia.org/wiki/Health_care_in_Canada.

[14] Canada, H. Canada's Food Guides. 2007 30/12/2013]; Available from: http://www.hc-sc.gc.ca/fn-an/food-guidealiment/context/fg history-histoire_ga-eng.php.

[15] Ontario, D.o.C.a.E., Eating Guidelines for Lacto-Ovo Vegetarians. 2009.

[16] association, A.p. 2013 23/5/2013 ; Available from: http://www.planning.org/nationalcenters/health/food.htm.

[17] Health care in Australia. 2013 4/1/2013]; Available from: http://en.wikipedia.org/wiki/Health_care_in_Australia.

[18] Faichney, G.J., A BRIEF HISTORY OF THE NUTRITION SOCIETY OF AUSTRALIA. 2006

[19] Dietitians of Canada and EatRight Ontario "Eating Guidelines for Lacto-Ovo Vegetarians". 2009.

[20] Misner, S., C. Curtis, and E.W. er, Vegetarian Diets Build On The Basics. 2008.

[21] CNA, Health Canada announces $\$ 8.1$ million to support portal 13 N. Canadian Nurse, 2006. 102: p. 1-9.

[22] Shim, J.P. and W.E. Sorrells, E-Health Technology Strategies and Impacts Case. E-HEALTH CARE INFORMATION SYSTEMS, 2005: p. 396.

[23] Torabi, M., A. Zamani, and B. Mahmoodzadeh, Building enterprise portals for knowledge-based, integrated, patientcentered healthcare systems in Iran, in Fourth EMRO regional conference of EHealth2004: Tehran·Iran. 